BNL-77930-2007-CP

\title{
Three-Dimensional Field Models for Reverse Biased P-N Junctions
}

F Ubaldi, G Pozzi, P F Fazzini ${ }^{1}$ and M Beleggia ${ }^{2}$

Physics Department and CNISM, University of Bologna, V.le Berti Pichat 6/2 - 40127 - Bologna - Italy

${ }^{1}$ CEMES-CNRS, 29 rue Jeanne Marvig, Toulouse Cedex 4 - 31055 - France

${ }^{2}$ Brookhaven National Laboratory, Building 480, Upton, NY 11973, USA

Presented at Microscopy of Semiconducting Materials

Cambridge, UK

April 2-5, 2007

April 2007

\section{Center for Functional Nanomaterials}

\author{
Brookhaven National Laboratory \\ P.O. Box 5000 \\ Upton, NY 11973-5000 \\ www.bnl.gov
}

Notice: This manuscript has been authored by employees of Brookhaven Science Associates, LLC under Contract No. DE-AC02-98CH10886 with the U.S. Department of Energy. The publisher by accepting the manuscript for publication acknowledges that the United States Government retains a non-exclusive, paid-up, irrevocable, world-wide license to publish or reproduce the published form of this manuscript, or allow others to do so, for United States Government purposes. 


\section{DISCLAIMER}

This report was prepared as an account of work sponsored by an agency of the United States Government. Neither the United States Government nor any agency thereof, nor any of their employees, nor any of their contractors, subcontractors, or their employees, makes any warranty, express or implied, or assumes any legal liability or responsibility for the accuracy, completeness, or any third party's use or the results of such use of any information, apparatus, product, or process disclosed, or represents that its use would not infringe privately owned rights. Reference herein to any specific commercial product, process, or service by trade name, trademark, manufacturer, or otherwise, does not necessarily constitute or imply its endorsement, recommendation, or favoring by the United States Government or any agency thereof or its contractors or subcontractors. The views and opinions of authors expressed herein do not necessarily state or reflect those of the United States Government or any agency thereof. 


\title{
Three-Dimensional Field Models for Reverse Biased P-N Junctions.
}

\author{
F Ubaldi, G Pozzi, P F Fazzini ${ }^{1}$ and M Beleggia ${ }^{2}$ \\ Physics Department and CNISM, University of Bologna, V.le Berti Pichat 6/2 - 40127 - Bologna \\ - Italy \\ ${ }^{1}$ CEMES-CNRS, 29 rue Jeanne Marvig, Toulouse Cedex 4 - 31055 - France \\ ${ }^{2}$ Brookhaven National Laboratory, Building 480, Upton, NY 11973, USA
}

Summary: In order to obtain reliable quantitative information on the electrostatic field associated with reverse-biased p-n junctions and on the distribution of dopants, the physics of the so-called "dead layer" and the influence of charged oxide layers are of paramount importance. To this purpose, experimental observations near the edge of a TEM sample can be useful. In these conditions, however, phase computations required to interpret the experimental results are very challenging as the problem is intrinsically three-dimensional. In order to cope with this problem, a mixed analytical-numerical approach is presented and discussed.

\section{Introduction}

Electron holography is a very powerful method for investigating semiconductor devices [1] and reverse-biased p-n junctions [2] at sub-10 $\mathrm{nm}$ resolution by means of transmission electron microscopy (TEM) techniques. However, these experiments are very challenging from the theoretical point of view. In fact, the simple classical model for describing the electrical behaviour of the reverse-biased p-n junction is no longer valid, and software packages like the ISE-tCad suite [3] are required to develop realistic models taking into account unexpected experimental effects like the charging-up of the dielectric layer [4,5].

In order to cope with this problem in the two-dimensional case, we use a mixed numericalanalytical approach in order to take into account the effect of the external fringing field without wasting computational resources. Recently, we have also developed a suitable analytical solution for the three-dimensional problem of an array of reverse-biased p-n junctions in a very thin specimen [6,7]. We are now investigating whether the numerical-analytical approach can be applied in the three dimensional case, in order to have a reliable model of the external field and to interpret edge-on observations.

\section{Numerical vs analytical calculations for a step junction}

With a finite-element numerical sofware package, the only way to assess its reliability is to vary the parameters until a stationary result is obtained within some preassigned error. In our case, the availability of an analytical model allows us to reduce the arbitrariness and to evaluate better the accuracy of the numerical approach. Therefore, we have first applied the ISE-tCad suite to simulate the case of an abrupt p-n junction in a very thin specimen and compared the results with the analytical calculations for the field and the integrated potential (proportional to the electron optical phase shift, which is the important quantity for the simulations of TEM observations). To this end, we extended the simulation domain independently in $z$ and $y$, and increased the sampling density across the junction until the electrostatic and the integrated potential showed negligible modifications in the region of interest (Fig. 1). The variation along the $x$ direction is not considered because it is directly related to the periodicity of the array. 


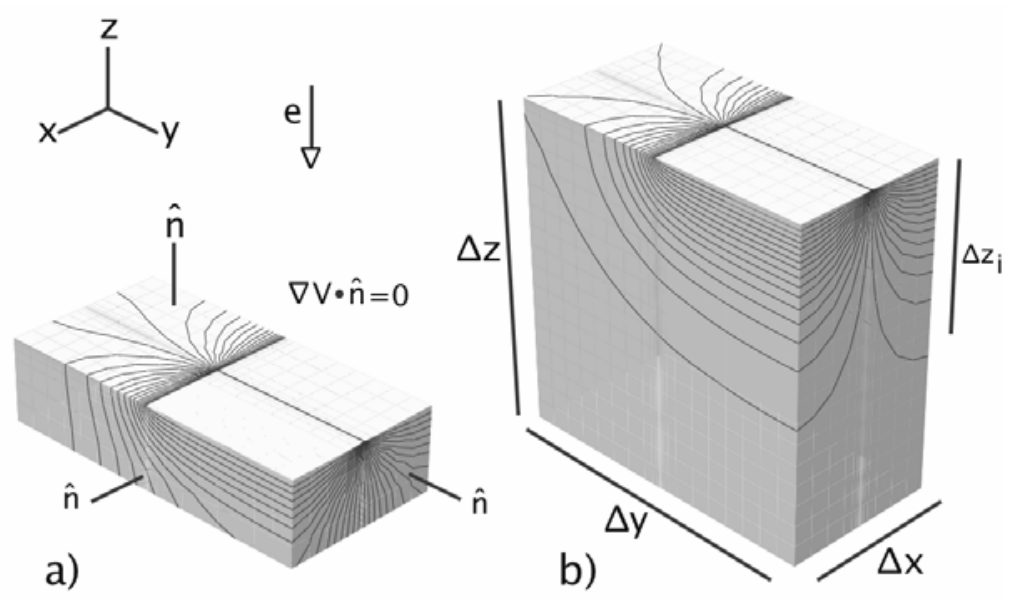

Fig. 1. Equipotential surfaces of a step junction $( \pm 1 \quad$ V) with negligible thickness; $\Delta x=4 \mu \mathrm{m}, \Delta y=8$ $\mu \mathrm{m}$. a) $\Delta z=2 \mu \mathrm{m}$; the simulation domain is too small along $z$, and artifacts introduced by the boundary condition $\nabla V \cdot \hat{\mathbf{n}}=0$ are evident when the potential topography is compared to $b)$ where the simulation domain was extended to $\Delta \mathrm{z}=8 \mu \mathrm{m}$ : in this case, the electrostatic potential is well contained in the simulation domain, vanishing before $z$ reaches the domain edge. Note also that the choice of $\Delta z$ affects the equipotential surfaces also along $y$, as visible by comparing the stray field potentials in the vacuum region $(y<0)$ of the junction plane $z=0$. In b) $\Delta z_{\mathrm{i}}$ denote the range of the integration of the potential used to evaluate the phase shift associated with the junction.

The comparison of numerical results with those obtained by calculating the analytical expressions through Mathematica [8] shows that we determined the appropriate domain size and sampling resulting in a maximum relative error of 0.001 in the potential and of 0.02 in the integrated potential calculated over the whole vertical length (Fig. 2), where the phase shifts are calculated for $200 \mathrm{keV}$ electrons.

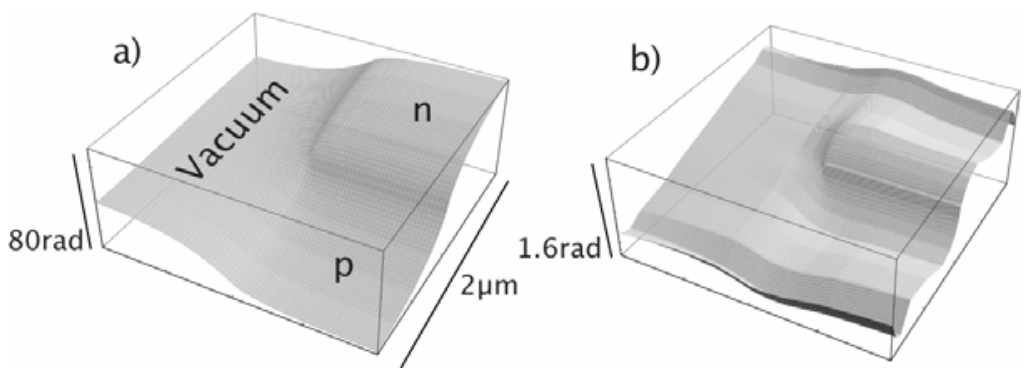

Fig. 2. a) Phase shift obtained by numerical integration along $z$ of the ISE-tCad potential of a -1 $\mathrm{V} \quad(\mathrm{p})+1 \quad \mathrm{~V} \quad(\mathrm{n})$ step junction. b) its difference with the analytical phase shift of the same junction.

The absolute difference decreases down to 0.8 rad by adjusting the range of integration $\Delta z_{i}$ to half $\Delta z$ (see Fig. 1).

It is interesting to note that this error decreases to 0.01 if the potential is integrated only up to half the simulation domain $\Delta z$. This effect is due to the distortion of the electrostatic potential near the simulation edges induced by the boundary conditions used in the numerical computation. These results show that the numerical evaluation of the phase shift is affected by the choice of the boundaries more strongly than the potential and that in order to have reliable results we have to "waste" numerical resources to include the empty space around the specimen. These resources may be better utilized in the device simulation if the external field and phase shift are calculated using the analytical results, whenever possible.

Since, however, real specimens are most likely thick objects, we have to account for their finite thickness. To this end, we employ a mixed numerical-analytical approach that consists in taking the analytical model for the zero-thickness step junction (Fig. 3a) and prolonging its $z=0$ plane potential along $z$ over the thickness $t$. This approximate potential is then compared with the 
fully 3D model (Fig. 3b): the equipotential lines look very similar for both cases and the comparison between the phase shifts confirms that the "prolonged" model is affected by a maximal relative error of about 0.04 . The error, however, lies mainly outside the region of interest across the junction.
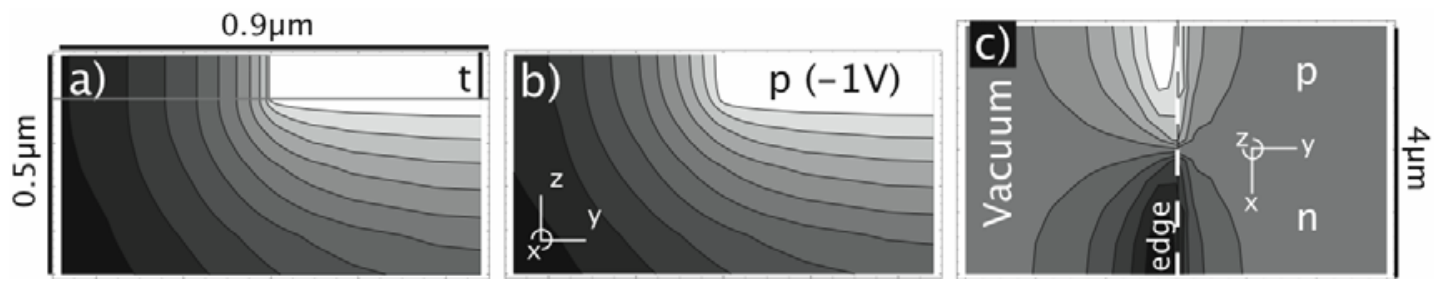

Fig. 3. a) Vertical section of the potential at $0.5 \mu \mathrm{m}$ from a $200 \mathrm{~nm}$ thick junction obtained by prolonging the $\mathrm{z}=0$ plane potential along the specimen thickness. This procedure results in straight equipotential lines over the thickness $t$. b) the fully $3 \mathrm{D}$ simulation over the same region, showing small differences with respect to a). c) phase difference contours between the models a) and b); the largest error is of $2.5 \%$, and becomes $4 \%$ at $300 \mathrm{~nm}$ thick. However, the error lies mainly outside the region of interest, across the junction.

\section{The CPAC model for the semiconductor junction}

To improve further our 3D model, we extend the former considerations to a realistic semiconductor junction, and introduce the CPAC model (Cut, Paste, Analytical Computation). This approach consists in building a 3D potential by cutting, pasting and prolonging with analytical computations parts of a two-dimensional (2D) potential cuts of a 2D simulation. The way of cutting, pasting and prolonging the potential is suggested by the similarity between the "prolonged" potential, as discussed above, and the fully 3D simulated potential. First, a 2D simulation is taken (Fig. 4a), and its surface potential is utilized to compute the potential in the region 3 of Fig. $4 \mathrm{~b}$ by using the analytical formula [1], while the inner potential is cut and pasted with a 45 degrees pattern to recover the $3 \mathrm{D}$ specimen potential. The choice of this particular patching angle $\left(45^{\circ}\right)$ is somewhat arbitrary, and most likely depends on the thickness. In fact, while it is certainly reasonable that in the limiting case when the specimen thickness is very large the symmetry dictates the same potential topography as a function of $y$ and $z$, for thin specimens it is conceivable that other patching angles may be more appropriate. Also, it is not clear whether patching should occur along a straight line rather than along a more general curve. However, for very thin specimens, the smaller relative contribution of the internal field topography to the total potential may minimize the effects of choosing a different patching procedure. Finally, the analytical potential of region 3 is extended over the thickness in front of the specimen with a constant $z$ prolongation to recover the potential of the region 4 .

Such a model is a good approximation for a real device if the full 3D surface potential doesn't vary appreciably in the directions orthogonal to the junction, i.e. $y$ and $z$. Fig. $4 c$ shows the equipotential lines of a 3D numerical simulations: the condition of constant surface potential along the $y$ and $z$ directions is satisfied. The comparison with the corresponding CPAC simulations (Fig. 4d) shows that the two models look very similar: the relative differences between phase shifts do not exceed 0.03 over the specimen (Fig. 4f).

\section{Conclusions}

We have described several steps towards the development of a realiable three-dimensional model for the electrostatic potential and electron-optical phase shift associated to a $p-n$ junction. The availability of a relatively simple analytical model for an ideal junction of negligible thickness is the building block of the realistic 3D model which is constructed from suitably cutting, pasting and 
prolonging two-dimensional slices of the potential. Since the CPAC model works rather satisfactorily for the thick abrupt and for the "ideal" thick semiconductor junction, we plan to extend it to cover even more realistic cases such as specimens coated with native surface oxides and electrically inactive layers.

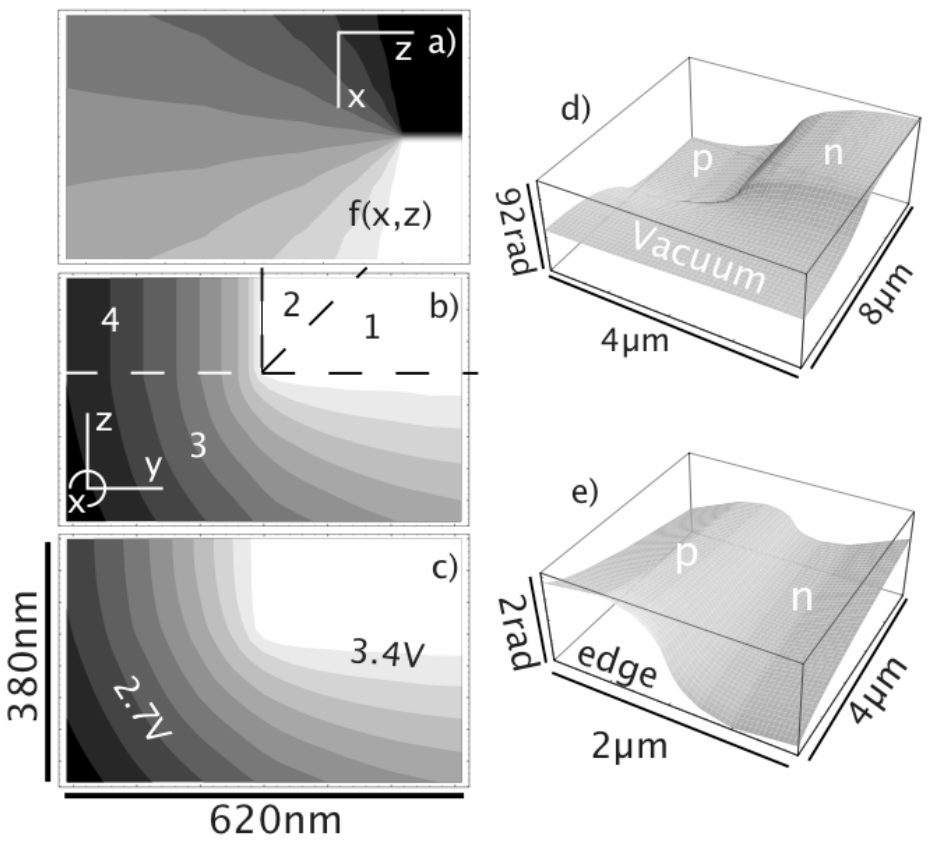

Fig. 4. The CPAC model of a $\mathrm{Sb}-\mathrm{B}$ junction at $3 \mathrm{~V}$ reverse bias: a) $f(x, z)$ is the $2 \mathrm{D}$ potential. b) the CPAC $3 \mathrm{D}$ model; region 1: the potential is $V(x, y, z)=f(x, z)$; region 2 : $V(x, y, z)=f(x, y) ; \quad$ region 3 : analytical computation from the 2D surface line; region 4: constant prolongation from region 3. c) numerical $3 \mathrm{D}$ model to be compared with the CPAC. d) phase shift associated to the numerical 3D model. e) phase difference between the two models over the specimen: the relative error is less than $3 \%$.

\section{Acknowledgements}

Financial support was provided by MIUR, FIRB funding RBAU01M97L, and by the U.S. Department of Energy, Basic Energy Sciences, under contract number DE-AC02-98CH10886.

\section{References}

1. Rau W D, Schwander P, Baumann F H, Hoppner W, Ourmazd A 1999 Phys Rev Lett 82, 2614 2617

2. Twitchett A C, Dunin-Borkowski R E, Hallifax R J, Broom R F, Midgley P A, 2005 Microsc.

Microanal. 11, 1-13, 2005

3. ISE Tcad v. 8.0 by Integrated System Engineering: http://www.ise.ch

4. Beleggia M, Fazzini P F, Merli P G, Pozzi G 2003 Phys. Rev. B 67, 045328 (2003)

5. Fazzini P F, Merli P G, Pozzi G, Ubaldi F 2005 Phys. Rev. B 72, 085312 (2005)

6. Beleggia M, Fazzini P F, Pozzi G 2003 Ultramicroscopy 96 (2003) 93-103

7. Fazzini P F, Pozzi G, Beleggia M 2005 Ultramicroscopy 104 (2005) 193-205

8. S. Wolfram. The Mathematica book. Product web page: http://documents.wolfram.com/v5/ 